\title{
MEJORAMIENTO GENÉTICO Y TAZA DE AUTOFECUNDACIÓN DEL CAMU CAMU ARBUSTIVO EN LA AMAZONÍA PERUANA ${ }^{1}$
}

\author{
CARLOS OLIVACRUZ² \& MARCOS DEON VILELADE RESENDE ${ }^{3}$
}

RESUMEN - El camu camu (Myrciaria dubia (H.B.K.) MC VAUGH) es una frutera silvestre conocida mundialmente como un excepcional productor de vitamina C. Su mejoramiento se encuentra en fase inicial. Este trabajo tuvo por objetivos estudiar el sistema reproductivo (taza de autofecundación), el efecto del origen del polen (autofecundación o polinización abierta) en la producción de ácido ascórbico y porcentaje de germinación, la repetibilidad de caracteres productivos y sus implicaciones en el programa de mejoramiento. El sistema reproductivo del camu camu es mixto con variables tazas de autofecundación. No fue confirmada la existencia de efecto del origen del polen para el carácter de producción de ácido ascórbico. La repetibilidad individual de la producción fue de moderada magnitud (0.41); la repetibilidad del promedio de 5 cosechas de fruta fue de 0.77 , propiciando exactitud selectiva de 0.88 . Genotipos superiores pueden ser seleccionados con precisión y, por lo tanto, cinco a seis cosechas por planta es un número adecuado. La selección y clonación de los diez mejores individuos deberá propiciar una ganancia genética del $237.5 \%$, elevando la productividad media anual de frutas por planta de 7.75 para $26.17 \mathrm{~kg} /$ año.

Términos para indexación: genética cuantitativa, repetibilidad, autofecundación, productividad.

\section{GENETIC IMPROVEMENT AND MATING SYSTEM OF THE CAMU CAMU SHRUB IN THE PERUVIAN AMAZON}

\begin{abstract}
The camu camu (Myrciaria dubia H.B.K. MC VAUGH) is a well known fruit species with a high vitamin C content. It is wild and a genetic improvement program is at an early stage. This paper reports on studies of its mating system, the possible effect of the source of the pollen (self pollination or outcrossing) and the repeatability of vitamin C production in this species with implications for the genetic improvement program. The results showed a mixed mating system with both crossing and selfing, no effects of the source of the pollen, individual repeatability of 0.41 , repeatability of the mean of five harvests of 0.77 and accuracy of 0.88 . Five to six fruit harvests is an adequate number to provide high selection efficiency. Selection and cloning of the best 10 trees can provide a genetic gain of $237.5 \%$, enhancing annual fruit production from 7.75 to $23.17 \mathrm{~kg}$ per plant.
\end{abstract}

Index terms: quantitative genetics, repeatability, self pollination, yield.

\section{INTRODUCCIÓN}

El camu camu (Myrciaria dubia H.B.K. MC VAUGH) es una especie conocida mundialmente como un excepcional productor de ácido ascórbico, comúnmente llamado vitamina $\mathrm{C}$, además contiene altos niveles de calcio y otros minerales de importancia bioquímica. Se distribuye en las cuencas de los ríos Amazonas y Ucayali, lugar donde ha desarrollado gran adaptación a las inundaciones. Tiene flores hermafroditas con alto porcentaje de polinización cruzada y reducido nivel de autogamia; a ello se debe la alta variabilidad fenotipica cualitativa y cuantitativa en esta especie. Es una especie silvestre en proceso de domesticación, y, según Vásquez (2000), tiene 91\% de alogamia y $9 \%$ de autogamia.

El camu camu pertenece a la familia Myrtaceae, puede alcanzar 6 a 8 metros de altura y presenta tres tipos de arquitectura: tipo columnar (poca o nula ramificación), tipo intermedio (ramificación inicia a los 50 a $70 \mathrm{~cm}$ del nivel del suelo) y coposa (ramificación desde el nível del suelo formando una gran copa). Las hojas son simples opuestas de forma lanceolada y plantas adultas miden de 3 a $6 \mathrm{~cm}$ de largo por 1,5 a 2,5 cm de ancho. Las flores son simples y nacen de las axilas de las hojas. Son hermafroditas y la antesis ocurre temprano por la mañana y las flores están receptibles a la polinización por un período de cuatro hasta cinco horas (Imán, 2001).

El mejoramiento de la especie se encuentra en fase inicial. En Brasil, los trabajos de recolección de germoplasma, conservación y mejoramiento son realizados por el Embrapa Amazonia Ocidental (Sousa \& Souza, 2001), Embrapa Amazonía Oriental (Silva, 1999) e Instituto Nacional de Pesquisas da Amazônia (INPA). En Peru, el programa de mejoramiento fue delineado por el Instituto Nacional da Amazonía Peruana (IIAP) y los estudios genéticos básicos se encuentran en desarrollo (Pinedo et al, 2004).

Este trabajo tiene por objetivos: estudiar el sistema reproductivo (taza de autofecundación) en la especie; estudiar el efecto del origen del polen en las características fenotípicas del fruto y de las semillas producidas por las plantas madres en la producción de ácido ascórbico y porcentaje de germinación; estudiar la repetibilidad de caracteres productivos y sus implicaciones en el programa de mejoramiento de la especie. Esto

'(Trabalho 129-07). Recebido em:18-05-2007. Aceito para publicação em: 14-12-2007.

2Ingeniero Agrónomo e Investigador en mejoramiento genético en el Instituto de Investigaciones de la Amazonia Peruana (IIAP) - coliva@iiap.org.pe. ${ }^{3}$ Ingeniero Agrónomo e Investigador en mejoramiento genético en Embrapa Florestas - deon@cnpf.embrapa.br. 
aparenta ser el primer trabajo a abordar la genética y mejoramiento de la especie con base en análisis de datos.

\section{MATERIALES Y MÉTODOS}

\section{Taza de Autofecundación}

En 2004, se realizaron ensayos de auto polinización en seis plantas de camu camu (Tabla 1), utilizando la técnica de aislar ramas con flores bisexuales para impedir el ingreso de polen extraño e inducir la autofecundación. El aislamiento se realizó con el empleo de bolsas de papel manteca poco antes del inicio de la apertura floral. Para tener un buen control en los resultados, se identificó para cada rama embolsada (autogamia) una rama testigo (alogamia); en estas ramas se realizó similar procedimiento diferenciándose de las anteriores por la ausencia de bolsas. Las bolsas se retiraron cuando las flores tomaron un color pardo (3 días después de la apertura), para que luego se evaluara a los 15 días en donde se cuantificó frutos pequeños similares a la cabeza de un alfiler.

Efecto del origen del polen (autofecundación o polinización abierta) en la producción de ácido ascórbico y porcentaje de germinación.

El efecto del origen del polen como el resultado de la fertilización que se manifiesta en los frutos o semillas de la planta madre ocurre en muchas plantas (Mercer et al., 2002) y puede ocurrir también en camu camu.

Usando las mismas plantas, se compararon la producción de ácido ascórbico y el porcentaje de germinación en frutos obtenidos por auto polinización y por polinización libre. Para ello se realizó la cosecha de los frutos en estado pinto maduro. Las muestras de fruta ingresaron al Laboratorio de Bioindustrias del IIAP-Ucayali, donde se realizó la selección, lavado, pulpeado, pesado (100 g de pulpa por muestra) y congelado $\left(-20 \mathrm{C}^{\circ}\right)$, y luego enviadas al Laboratorio de Ingeniería Química de la Universidad Nacional de la Amazonia Peruana, para su análisis mediante el método de titulación 2-6 diclorofenlindolfenol. Las semillas fueron seleccionadas e instaladas en las camas para su germinación, utilizando como sustrato la arena y con riego dos veces al día, conforme metodología empleada por IIAP.

\section{Repetibilidad de la producción de frutas.}

Se evaluaron 315 plantas seleccionadas, de procedencia de las cochas Sahua y Morona y del río Nanay, ubicadas en la Región de Lotero. Estas plantas están establecidas desde 1988 en el Anexo de Pacacocha-Ucayali, donde el INIEA-Pucallpa ha evaluado el rendimiento de la fruta en Kg-pl-año. La estación experimental está ubicada en suelos de la orden Entisols y Sub orden Fluvents, con pH cambiando de 6,4 a 7,6. El clima se caracteriza por ser cálido y húmedo con una temperatura media anual de 25,1 y rango de 17,4 a 36,5 grados Celsius. La precipitación media anual es de $1447,31 \mathrm{~mm}$. La matriz de datos de cinco cosechas consecutivas (1998 al 2002) fue analizada en programa genético computarizado Selegen-Reml/Blup (Resende, 2002), permitiendo conocer la repetibilidad, exactitud selectiva y ganancia genética con la selección de los caracteres de producción.

Los valores genotípicos de cada planta fueron estimados por, $\hat{g}=M G+\beta_{p}\left(M P_{i}-M G\right)$ en que:

$\beta_{p}=\frac{m \rho}{1+(m-1)}$ $m \hat{\rho}$

fenotípico permanente o repetibilidad de la media de $\mathrm{m}=5$ mediciones en el individuo;

$\hat{\rho}$ : estimativa de la repetibilidad individual.

$M G$ : media general de las plantas en varias mediciones; $M P_{i}$ : media general da la planta i en las varias mediciones. La ganancia genética fue estimada como el promedio de valores genotípicos de los individuos seleccionados.

\section{RESULTADOS Y DISCUSIONES}

\section{Taza de Autofecundación}

Después de 15 días, se obtuvo hasta el 96,5\% de autofecundación en la planta 169 , frente a $91,9 \%$ de polinización cruzada (tratamiento testigo) en la misma planta. Por otro lado, los menores resultados de autofecundación se reflejan en la planta 264 con el 7,3\% y 50,7\% de polinización libre (Tabla 1). Esta variación se considera natural pues esta especie presenta sincronismo entre viabilidad del polen y la receptabilidad del estigma por un periodo de 60 a 90 minutos la cual condice a la existencia de dicogamia que actúa como limitante para la autofecundación en esta especie (Maues \& Couturrier, 2002). En el eucalipto la autofecundación está reducida por el hecho de que los tubos polínicos del polen extraño crecen relativamente rápidos en el estigma y tienen por lo tanto una mayor probabilidad de fecundar el óvulo (Eldridge et al., 1993).

Los resultados demuestran que el camu camu tiene singular comportamiento reproductivo que varia de una planta a otra. La mayor dispersión de datos se encuentra en la autofecundación (autogamia) con valores que van desde 7,3 hasta $96,5 \%$, sin embargo en la polinización libre (alogamia) es mas homogéneo desde 39,0 hasta 91,9\% (Tabla 1). En la polinización libre, la fertilización está influenciada por diferentes factores, principalmente insectos (atraídos por el aroma que expiden las flores) y el viento, permitiendo de esta manera mayor efectividad por efecto de la alta competencia entre polen de diversas procedencias. Los resultados de autofecundación están de acuerdo en parte con lo reportado por Vásquez (2000), pero probablemente el autor haya realizado sus experimentos en una planta con características similares a las plantas 264,186 y 31 , que son plantas con reducido porcentaje de autofecundación. Sistema reproductivo con variación de planta a planta en tazas de autofecundación en cada planta son también típicos de otras mirtáceas como la guayaba (Soubihe Sobrinho \& Gurgel, 1962, Pereira \& Nachtigal, 2002) y el eucalipto (Eldridge et al., 1993).

El promedio de autogamia es $28,107 \%$ y alogamia 59,798 $\%$, como se observa, la desviación estándar en autogamia supera al promedio siendo así más variable (Tabla 2). Se observa que cerca del $50 \%$ de botones florales no son fecundados a pesar de ser la polinización libre y existir más factores favorables, 
definitivamente el porcentaje de alogamia es superior debido a que las especies han desarrollado muchos mecanismos que promueven nuevas combinaciones genéticas, las cuales incluyen alelos de autoesterilidad y de adaptaciones anatómicas que inhiben la autofecundación (Richards, 1986).

Con relación a la metodología usada para estudiar la taza de autofecundación es importante mencionar que el uso de progenies y marcadores genéticos es una herramienta más precisa y debe ser utilizada para confirmación de los resultados. ascórbico.

Efecto del origen del polen en la producción de ácido

Con relación al contenido de ácido ascórbico, las muestras provenientes de polinización libre (alogamia) tienen mayor contenido de ácido ascórbico en todas las muestras, y cuyas diferencias no son significativas con relación al contenido de ácido ascórbico en frutos autofecundados (Tabla 2).

Los valores medios obtenidos en los frutos cruzados fue 1548 y en los autofecundados fue 1368 (Tabla 2). Esa diferencia no es significativa conforme verificado por los desvíos patrones, pero puede existir efecto del origen del polen (xenia y endogamia) para el carácter producción de ácido ascórbico, con polen de la misma planta causando un decrecimiento en el contenido. Nuevos estudios con mayores tamaños de muestra son necesarios. La presencia del efecto de Xenia ha sido verificada en varias especies vegetales como el maíz (Mercer et al., 2002), donde se ha reducido el peso de semillas y el contenido de ciertos compuestos en semillas autofecundadas.

Efecto del origen del polen en la viabilidad de semillas.

Además del contenido de ácido ascórbico se observó que las semillas provenientes de frutos autofecundados tuvieron menor porcentaje de germinación, a diferencia de la planta 169 quien logró el 100\% de germinación en los dos casos alogamia y autogamia. El comportamiento en el porcentaje de germinación es independiente de una planta a otra reflejando variabilidad intra específica. Sarvas (1962) informa que existe mortalidad de embriones en Pinus sylvestris por efecto de la autofecundación pero no necesariamente debido a xenia, a ello espaldan Ehrenberg et al. (1955) que la autofecundación da lugar a germinación pobre en semillas desarrolladas y a descendencias inferiores. Es importante destacar que los efectos de la xenia y de la endogamia son asociados a conceptos diferentes.

Se encuentra un promedio del $66,33 \%$ de germinación en frutos autofecundados y $91,33 \%$ de germinación en frutos cruzados (Tabla 2). En otras especies de la misma familia, por ejemplo en Eucalyptus, el porcentaje de plántulas autofecundadas disminuirá aun más por el frecuente bajo vigor de las plántulas intra cruzadas (Hodgson, 1974, van Wyk, 1981).

\section{Repetibilidad de la producción de frutas}

La repetibilidad individual de la producción de frutas fue de 0,41 y la repetibilidad del promedio de 5 cosechas fue de 0,77 . Asi, la selección basada en este promedio propicia exactitud selectiva de 0,88 (Tablas 3 y 4). De ese modo, los genotipos superiores pueden ser seleccionados con precisión.
La repetibilidad se refiere a la correlación entre medidas repetidas realizadas en un mismo individuo. La magnitud observada $(0,41)$ en este trabajo es considerada moderada (Resende, 2002). A partir de esta estimación se puede inferir que la heredabilidad del carácter producción es baja a mediana porque la repetibilidad representa el valor máximo que la heredabilidad puede asumir.

Para evaluaciones futuras en el programa de mejoramiento, el número ideal de mediciones por planta puede ser determinado, en función de la exactitud de la selección y la determinación (Tabla 4). Deseando una exactitud del $90 \%$ en la selección, o sea, $80 \%$ de determinación, indica que se deben evaluar 6 cosechas por planta. Esto propicia una confiabilidad selectiva del $90 \%$, la cual es adecuada para el propósito del mejoramiento genético. Propicia también una eficiencia de 1,41 (superioridad de 41\%) con relación al uso de solo una cosecha.

\section{Selección Genética para Producción de Frutas}

Los resultados de la selección genética con base en cinco cosechas revelan un alto potencial para obtener ganancia genética en esta población (Tabla 5). La selección y clonación de los 10 mejores individuos deberá propiciar una ganancia genética de $237,5 \%$, elevando la productividad media anual por planta de $7,75 \mathrm{~kg} /$ año para 26,17 kg/año (Tabla 5). Esto es debido al hecho de que la especie es aún silvestre, con amplia variabilidad genética disponible para selección y mejoramiento. Así, programas de mejoramiento genético de camu camu son muy estratégicos y esenciales para el desarrollo del sector productivo asociado a esta especie.

Ganancias mayores podrían obtenerse con la selección de las cinco mejores plantas. Pero un número muy pequeño de plantas madres podría causar vulnerabilidad de los plantíos comerciales oriundos de ellas. Eso es debido a la baja variabilidad genética asociada a un pequeño tamaño efectivo poblacional.

TABLA 1- Comportamiento del número de botones florales cuanto al porcentaje de fertilización cruzada y autogamia en las plantas de camu camu, en el Anexo de Pacacocha - Peru.

\begin{tabular}{crrrr}
\hline Código & \multicolumn{2}{c}{ Número de Botones Florales } & \multicolumn{2}{c}{ Porcentaje de Fertilización } \\
\cline { 2 - 5 } Planta & Autogamia & Alogamia & Autogamia & Alogamia \\
\hline 252 & 854,0 & 510,0 & 20,06 & 53,56 \\
264 & 1169,0 & 708,0 & 7,29 & 50,68 \\
186 & 594,0 & 393,0 & 8,66 & 48,16 \\
31 & 723,0 & 563,0 & 12,61 & 39,03 \\
293 & 988,0 & 741,0 & 23,49 & 75,44 \\
169 & 377,0 & 91,0 & 96,53 & 91,92 \\
\hline Promedio & 784,17 & 501,00 & 28,11 & 59,80 \\
\hline Desvío & - & - & 34,12 & 19,83 \\
Padrón & & & & - \\
\hline Total & 4705,0 & 3006,0 & & - \\
\hline
\end{tabular}


TABLA 2- Comportamiento del contenido de ácido ascórbico y porcentaje de germinación, en función del porcentaje de fertilización cruzada y autogamia en las plantas de camu camu, en el Anexo de Pacacocha - Peru.

\begin{tabular}{crrrr}
\hline Código & \multicolumn{2}{c}{ Contenido de Ácido Ascórbico } & \multicolumn{2}{c}{ Porcentaje de Germinación } \\
\cline { 2 - 5 } Planta & Autogamia & Alogamia & Autogamia & Alogamia \\
\hline 252 & 1480,90 & 1628,25 & 47,50 & 80,00 \\
264 & 1286,50 & 1333,41 & - & - \\
186 & 1573,16 & 1633,70 & 42,86 & 92,86 \\
31 & 768,37 & 1412,54 & 75,00 & 92,50 \\
293 & 1243,84 & 1326,55 & - & - \\
169 & 1857,64 & 1957,69 & 100,00 & 100,00 \\
\hline Promedio & 1368,40 & 1548,69 & 66,34 & 91,34 \\
\hline Desví & 367,75 & 242,93 & 26,55 & 8,31 \\
Padrón & & & & \\
\hline
\end{tabular}

TABLA 3 - Componentes de varianza asociados a la producción de frutas en plantas de camu camu, en el Anexo de Pacacocha - Peru, estimados por máxima verosimilitud restringida (REML).

\begin{tabular}{cl}
\hline Varianza fenotípica permanente & 39,44 \\
Varianza ambiental temporaria & 57,52 \\
Varianza fenotípica individual & 96,96 \\
Repetibilidad individual & $0,41 \pm 0,05$ \\
Repetibilidad de media de cosechas & 0,77 \\
Exactitud selectiva & 0,88 \\
\hline Media general & 7,75 \\
\hline
\end{tabular}

TABLA 4 - Coeficiente de determinación, exactitud selectiva y eficiencia del uso de $m$ mediciones repetidas en vez de una, asociados a la producción de frutas en plantas de camu camu, en el Anexo de Pacacocha - Peru.

\begin{tabular}{|c|c|c|c|}
\hline $\begin{array}{c}\text { Número de } \\
\text { mediciones }(\mathrm{m})\end{array}$ & Determinación & $\begin{array}{l}\text { Exactitud } \\
\text { selectiva }\end{array}$ & Eficiencia de m mediciones \\
\hline 1 & 0,4067 & 0,6378 & 1,0000 \\
\hline 2 & 0,5783 & 0,7604 & 1,1924 \\
\hline 3 & 0,6729 & 0,8203 & 1,2862 \\
\hline 4 & 0,7328 & 0,8560 & 1,3422 \\
\hline 5 & 0,7742 & 0,8799 & 1,3796 \\
\hline $\mathbf{6}$ & $\mathbf{0 , 8 0 4 4}$ & $\mathbf{0 , 8 9 6 9}$ & $\mathbf{1 , 4 0 6 3}$ \\
\hline 7 & 0,8276 & 0,9097 & 1,4264 \\
\hline 8 & 0,8458 & 0,9197 & 1,4420 \\
\hline 9 & 0,8605 & 0,9277 & 1,4545 \\
\hline 10 & 0,8727 & 0,9342 & 1,4648 \\
\hline
\end{tabular}

TABLA 5 - Resultados de la selección genética asociados a la producción de frutas en plantas de camu camu, en el Anexo de Pacacocha - Peru, con base en cinco cosechas.

\begin{tabular}{|c|c|c|c|c|c|c|}
\hline Orden & Individuo & $\begin{array}{c}\text { Valor } \\
\text { Fenotípico } \\
(\mathrm{Kg} / \text { año })\end{array}$ & $\begin{array}{c}\text { Valor } \\
\text { Genotípico } \\
(\mathrm{Kg} / \text { año })\end{array}$ & $\begin{array}{c}\text { Ganancia } \\
\text { Genética } \\
(\mathrm{Kg} / \text { año })\end{array}$ & $\begin{array}{c}\text { Ganancia } \\
\text { Genética } \\
(\%)\end{array}$ & $\begin{array}{c}\text { Promédio de la } \\
\text { Población } \\
\text { Mejorada } \\
\text { (Kg/año })\end{array}$ \\
\hline 1 & 211 & 25,482 & 33,232 & 25,482 & 328,8 & 33,232 \\
\hline 2 & 253 & 23,432 & 31,182 & 24,457 & 315,6 & 32,207 \\
\hline 3 & 295 & 21,701 & 29,451 & 23,538 & 303,7 & 31,289 \\
\hline 4 & 293 & 21,311 & 29,062 & 22,981 & 296,5 & 30,732 \\
\hline 5 & 91 & 19,173 & 26,923 & 22,220 & 286,7 & 29,970 \\
\hline 6 & 43 & 15,152 & 22,903 & 21,042 & 271,5 & 28,792 \\
\hline 7 & 261 & 15,083 & 22,833 & 20,191 & 260,5 & 27,941 \\
\hline 8 & 84 & 14,665 & 22,415 & 19,499 & 251,6 & 27,250 \\
\hline 9 & 165 & 14,200 & 21,951 & 18,911 & 244,0 & 26,662 \\
\hline 10 & 169 & 13,980 & 21,730 & 18,418 & 237,5 & 26,168 \\
\hline
\end{tabular}

\section{CONCLUSIONES}

El sistema reproductivo del camu camu es mixto, con variables tazas de autofecundación dependiendo de las plantas y ambiente. Nuevos estudios son necesarios para verificar la existencia de efecto de xenia para el carácter de producción de ácido ascórbico, con polen de la misma planta causando un decrecimiento del contenido. Las semillas de frutos de polinización libre tienen mayor capacidad de germinación. La repetibilidad de la producción fue de moderada magnitud, mostrando que la heredabilidad de carácter es moderada. Para trabajos futuros se recomienda seleccionar con base en seis evaluaciones por planta. La selección y clonación de los diez mejores individuos deberá propiciar una ganancia genética importante, elevando la productividad media anual de frutas por planta.

\section{REFERENCIAS}

EHRENBERG, C.; GUSTAFSSON, A.; PLYM FORSHELL, C. P.; SIMAK, M. Seed quality and the principles of forest genetics. Hereditas, Lund, v.41, p. 291-366, 1955.

ELDRIDGE, K.; DAVIDSON, J.; HARWOOD, C.; WYK, G. van. Eucalypt domestication and breeding. Oxford: Oxford Science Publications. 1993, 288 p.

HODGSON, L.M. Breeding of Eucalyptus in South Africa. South African Forestry Journal, Johannesburg, v. 89, p.13-15, 1974. 
IMÁN, C.S. Cultivo de camu-camu Myrciaria dubia H.B.K. Mc Vaugh en la región de Loreto. Iquitos: Universidad Nacional de la Amazonía Peruana, 2001. 65p.

MAUES, M. M.; COUTURRIER, G. Biologia floral e fenologia reprodutiva do camu camu (Myrciria dubia (H.B.K.) Mc Vaugh) no Estado do Pará, Brasil. Revista Brasileira de Botânica, São Paulo, v. 25, n.4, p. 441-448, 2002.

MERCER, J.R.; RAMALHO, M.A.P.; RAPOSO, F.V. Implicações do fenômeno xênia nos programas de melhoramento de milho. Ciência e Agrotecnologia, Lavras, v.26, n.6, p.1338-1343, 2002.

PEREIRA, F.M; NACHTIGAL, J.C. Goiabeira. In: Bruckner, C.H. Melhoramento de fruteiras. Viçosa: Editora UFV, 2002. p.267289.

PINEDO, M.; LINARES, C.; MENDOZA, H.; YANGUIZ, R. Plan de Mejoramiento genético del camu camu arbustivo. Iquitos: Instituto de Investigaciones de la Amazonia Peruana, 2004. 52p.

RESENDE, M.D.V. Genética biométrica e estatística no melhoramento de plantas perenes. Brasília: Embrapa Informação Tecnológica, 2002.975p.

RICHARDS, A.J. Plant breeding systems. London: George Allen Publishers, $1986.529 \mathrm{p}$.
SARVAS, R. 1962. Investigations on the flowering and seed crop of Pinus sylvestris. Communicationes Instituti Forestalis Fennica, Helsinki, v.53, n.4, p. 1-198, 1962.

SILVA, J.F. Camu - camuzeiro (Myrciaria dúbia). In: EMBRAPA. Centro de Pesquisa Agroflorestal da Amazonia Oriental (Belem, PA). Programa de melhoramento genetico e de adaptacao de especies vegetais para a Amazonia Oriental. Belem: Embrapa Amazonia Oriental, 1999. p.25-27.

SOUBIHE SOBRINHO, J.; GURGEL, J.T.A. Taxa de panmixia na goiabeira. Bragantia, Campinas, v.21, n.2, p.15-20, 1962.

SOUSA, N. R. ; SOUZA, A. das G. C. de. Recursos Genéticos de camu-camu. In: Nelcimar Reis Sousa; Aparecida das Graças Claret de Souza. (Org.). Recursos fitogenéticos da Amazônia Ocidental. Manaus: Embrapa Amazônia Ocidental, 2001, p. 78-87.

VÁSQUEZ, M.; MATUTE A. El Camu camu: cultivo, manejo e investigaciones. Iquitos: Universidad Nacional de la Amazonía Peruana, 2000. 84p.

WYK, G. VAN. Inbreeding effects in Eucalyptus grandis in relation to degree of relatedness. South African Forestry Journal, Johannesburg, v. 116, p.60-63, 1981. 